\title{
Pedagogía crítica: Estrategia de SOCIALIZACIÓN DEL POSCONFLICTO EN LOS RESGUARDOS INDÍGENAS
}

\author{
CRITICAL PEDAGOGY: STRATEGY OF SOCIALIZATION OF \\ POSCONFLICT PROCESS IN THE INDIGENOUS REARSGUARD
}

Por: Alexander Gómez Flórez*

*Estudiante del Programa de Filosofía de

la Universidad de Car-

tagena $y$ de Derecho de

tagena y de Derecho de

la Universidad del s

seccional Cartagena,

integrante del Semillero

de Investigación Dere-

cho Educación y Acción

(Unilibre).

Recibido: 15 de marzo de 2016 - Aprobado: 26 de julio de 2016

\section{RESUMEN}

Colombia se encuentra en una reconstrucción de su tejido social a través del desarrollo del marco de las políticas públicas proyectadas en el gobierno de Juan Manuel Santos Calderón debido al avance positivo del Proceso de Paz en La Habana-Cuba, fortaleciendo en la praxis la libertad de pensamiento del nuevo ciudadano pluriétnico y su accionar en los resguardos indígenas, generando la nueva condición como ciudadano multicultural. Tal propedéutica involucra a la pedagogía crítica como estrategia dentro del proceso de resocialización y concertación del posconflicto, permitiendo ser interpretado desde diferentes asentamientos ideológicos principalmente en la educación humanista para poder develar y abordar: El posconflicto.

Palabras Claves: Indígenas, Multicultural, Cabildo, Pluriétnico, Posconflicto, Resguardos.

\begin{abstract}
Colombia is in a reconstruction of its social fabric, through the development of the framework of public policies projected in the government of Juan Manuel Santos Calderon, due to the positive development of the peace process in Havana, Cuba, strengthening in the practice of freedom of thought of the new multi-ethnic citizen, which increases their actions in the indigenous reservations, empowering their new condition as a multicultural citizen. Such propaedeutic involves critical pedagogy in a process of re-socialization and post-conflict reconciliation, allowing interpretation from different ideological settlements, such humanistic education, in order to uncover: The Post-conflict.
\end{abstract}

Keywords:Indigenous, multicultural, Council, Multi-ethnic, Post-conflict, Reservation.

\section{Introducción}

E n la actualidad los resguardos indígenas son escenarios de nuevas políticas públicas en el marco del posconflicto planteado en la mesa de negociaciones de La Habana-Cuba entre el Gobierno y los miembros de las FARC. Según la Encuesta Nacional (Gómez, 2012) indicó el porcentaje del $2 \%$ en el cual se enmarca la población indígena, carece de acciones 
que posibiliten la concreción de los DDHH y DIH, lo anterior, planteado psicosocialmente es un detonante del conflicto y escenario para analizar el desarrollo de estrategias para desarrollar las políticas públicas propias del posconflicto en los resguardos del Chocó, dentro este escenario se oferta y facilita a los grupos armados antes denominados "ilegales" acciones para la reinserción social, como escribe Bolaños (2016).

La población perteneciente a los resguardos Embera Katío, Embera Chamí y Embera Dobida, expresan el territorio Representa la Vida, es la Madre Naturaleza, es nuestra casa donde nos relacionamos armónicamente con todos los seres vivientes, con sus espíritus, y sus energías, por ese conocimiento tradicional heredado de nuestros ancestros, depende de esa relación íntima con todo lo que nos rodea para seguir existiendo. La educación juega un rol importante, no solo en impartir, sino en proteger la identidad cultural, el Estado Social y Democrático de Derecho tiene esta función además de propugnar por la vigencia de un principio de igualdad, fundamento de un Estado Constitucional.(Ulloa, 2016).

La Corte Constitucional (2009) en el auto 004 del 2009, establece la protección de los derechos fundamentales de las personas y pueblos indígenas afectados por el conflicto armado que vive Colombia, sí este auto no se cumple a cabalidad genera una disociación interna, lo cual constituye un elemento para analizar como en Chocó gran parte de la población indígena no tiene SISBEN, propendiendo un análisis y el fortalecimiento de planes de desarrollo enfocados a la educación y la salud como derechos fundamentales. El Sistema de Selección de Beneficiarios Para Programas Sociales (SISBEN) es una herramienta, conformada por un conjunto de reglas, normas y procedimientos para obtener información socioeconómica confiable y actualizada de grupos específicos en todos los departamentos, distritos y municipios del país. (Ramírez, 2015).

El SISBEN es la puerta de entrada al régimen subsidiado, busca focalizar el gasto público para garantizar que el gasto social sea asignado a los grupos de población más pobres y vulnerables; su objetivo central es establecer un mecanismo técnico, objetivo, equitativo y uniforme de selección de beneficiarios del gasto social para ser usado por las entidades territoriales en programas sociales y en las áreas de salud, educación, bienestar social, entre otras. Botero (2005, p.77) indicó lo relacionado con lo socioeconómico y lo académico, permite desarrollar estrategias mediante la pedagogía crítica posibilitando dimensionar ciudadanos políticos capaces de contrastar y 
generar la construcción de procesos concertados como ciudadano-víctimas, ciudadano-victimarios en relación con el Estado Social y Democrático de Derecho. (Sentencia C-288 del 2012).

En el campo educativo, la vulneración se observa por la falta de instituciones con las garantías mínimas constitucionales, no hay sinergia entre las políticas públicas del Estado con la realidad social, en contraste los resguardos Embera Katío y Embera Chamí crean un currículo, conteniendo en su modelo pedagógico el Proyecto Educativo Cultural Territorial Indígena en siglas PECTI, tratando de preservar el conocimiento tradicional heredado de sus ancestros (Caisamo, 2012). De esta manera, se deja planteado que desconocer el posconflicto desde el aspecto sociojurídico, socioeconómico, los asentamientos ideológicos filosóficos y la psicología social, no es un garante para posibilitar una respuesta concertada del conflicto, por tanto la invitación del documento es aproximarse a esta realidad desde los ítems demarcados.

\section{Metodología}

Tr a metodología de este documento es teórico-crítica se basa en fuentes primarias y secundarias. Las fuentes primarias utilizadas son el pensamiento de Freire, los puntos de discusión de la mesa de negociaciones, la agenda de paz del gobierno y documentos de memoria histórica y las leyes concomitantes que rigen el desarrollo de esta temática. Los secundarios son pensadores como Derrida y Foucault. El objetivo de este documento es plantear la inmersión de la pedagogía crítica como estrategia de socialización del posconflicto en los resguardos indígenas. Se intentará seguir un orden propositivo en cuantos a los diferentes asentamientos ideológicos para fortalecer el planteamiento pedagógico y su quehacer en el nuevo escenario de reconstrucción social.

\section{Asentamiento ideológico desde lo sociojurídico}

Tr a justicia transicional es un nuevo modelo de orden internacional que trastoca los elementos de la guerra en Colombia (históricamente Juna de las más largas y que sigue vigente). Este conflicto presenta escenarios particulares como las cifras de violencia, los falsos positivos por parte de las fuerzas armadas del Estado, genocidios cometidos por los paramilitares; la inclusión de niños en el conflicto de las FARC y el ELN, los cilindros bomba, las minas antipersona; en la actualidad sistemas híbridos entre estos, para proteger, el cultivo, el procesamiento y el mercadeo ilegal de drogas alucinógenas. 
Tal reconstrucción de los hechos permite tener un duelo y, uno de los factores más importantes entre víctimas, victimarios y el Estado, para la no repetición de tales hechos. Aunque las treguas "vacilantes e intermitentes" del Proceso de Paz no han apagado el impacto de los fusiles y el bombardeo en el nororiente de Antioquia y el bajo Cauca en la data del segundo semestre del 2015. En el ideal colectivo, tanto en la retina de los colombianos no importando sexo o edad se clama en cada oración por la paz y la esperanza de un futuro mejor.

Una estrategia para socializar los componentes del posconflicto es la pedagogía crítica, porque propone un alto impacto social comunicativo crítico desde adentro de la comunidad indígena, mediante el cuestionamiento de la realidad social creada por el cambio del estos en forma abrupta y violenta, esto ayuda a desafiar las fuerzas latentes que todavía surten en la justicia distributiva; concertando lo cultual y lo cultural como símbolo de sociedad e identidad como manera excelsa de patentar un concepto de comunidad organizada mediante la planeación participativa.

Como más adelante se planteará los vasos comunicantes entre Freire y Derrida, a partir de un logos dialogado, de una comunicación reconstruida, deconstruida por la necesidad misma de romper con los lazos occidentales que de una u otra manera presionan e invaden lo ancestral, tratando de formar unos resguardos más consolidados, instituciones sociales más comunicativas, y un tejido social idóneo, es en sí la estrategia. Por último, la familia será mejor pensadas, basadas y estructuradas en seres conscientes y críticos de su realidad sin desvirtuar sus raíces como parte de sus respuestas liberadoras tanto en lo básico como en lo estructural, otorgándole un mejor devenir al ciudadano político y multicultural.

\section{Asentamiento ideológico, aspectos socioeconómicos}

- a Unidad para la Atención y Reparación Integral de Victimas indicó que hasta el 2012 se registraron 152.312 hechos de desplazamiento en la Región Caribe. Las expectativas del proceso de paz que se emprendió con estas organizaciones parecen dar frutos actualmente. El comportamiento se mantuvo más o menos estable entre 2004 y 2008, cuando osciló entre 64.270 y 78.765 hechos de desplazamiento. La indemnización administrativa a las víctimas de desplazamiento forzado se reconocerá por hogar y por hasta 17 salarios mínimos mensuales legales vigentes. La ACNUR índico que en el Chocó tres generaciones de las mismas familias han sufrido desplazamiento interno, mostrando la realidad del desplazamiento y su relación con la pobreza. 
La Constitución (1991) en el artículo constitucional 286, acerca de los territorios indígenas como entidades territoriales de la nación es una clara definición para el nuevo ordenamiento territorial, y de ella se derivan consecuencias fundamentales para autonomía de los pueblos indígenas. Igualmente, en el artículo 329, inciso segundo, la constitución política dispone "los resguardo son de propiedad colectiva y no enajenable" y el parágrafo del artículo 330 establece la participación de las comunidades indígenas en las decisiones relacionadas con los recursos naturales en sus territorios. (p. 72).

La sentencia C-139/1996, determina la manera como debían ser gobernados los "salvajes" que vayan reduciéndose a la vida civilizada, proporcionando nuevos espacios de convivencia y legitimidad social del indígena. Consecuencia de lo anterior, el indio ya no es un salvaje como establecía la Ley 89 de 1890; por su parte el Decreto 1396 de 1996 por el cual se crea la Comisión Nacional de Territorios Indígenas y además el programa especial de atención a Pueblos Indígenas; a su vez el Decreto 1397 de 1996 crea la Comisión Nacional de Territorios Indígenas y la mesa permanente de concertación con los pueblos y las organizaciones indígenas, favorecen la generación de una nueva sociedad.

También, el Decreto 2164 de 1995 hito jurídico por brindarles la titulación de tierra a las comunidades indígenas; el Decreto 1320 de 1998 el cual reglamenta la consulta previa con las comunidades indígenas y negras para la explotación de recursos naturales dentro de su territorio; el Decreto 982 de 1999 crea la comisión para el desarrollo integral de la política indígena; la Ley 21 de 1991 aprueba el convenio 169 de la OIT; el Decreto 1088 de 1993 regula la creación de las asociaciones de cabildos-autoridades tradicionales indígenas; la Ley 160 de 1994 el Sistema Nacional de Reformas Agrarias y Desarrollo Rural y Campesino; el Decreto 804 de 1995 la atención educativa para las comunidades étnicos, la Ley 649 de 2001 la Circunscripción Nacional Especial y la ley 1381 del 2010 la Ley de lenguas, todas favorecen el proceso social-comunitario.

La indemnización que reciban los niños y adolescentes, se entregará a través de encargo fiduciario. Los niños y adolescentes recibirán efectivamente su indemnización cuando cumplan su mayoría de edad. Esta indemnización administrativa se entregará en el marco del programa de acompañamiento para la inversión adecuada de los recursos. Esto quiere decir que la Unidad para las Víctimas ofrecerá opciones de inversión de los recursos a las personas 
del hogar que recibirán la indemnización para contribuir a la toma de decisión sobre el uso de los recursos y que, la misma pueda contribuir a la reconstrucción del proyecto de vida del hogar. En todo caso, la participación en el programa de acompañamiento es voluntaria.

La Unión Europea ratificó su respaldo al proceso de paz con las Farc y anunció un nuevo aporte de 575 millones de euros para apoyar la implementación de los acuerdos que se firmen para acabar con más de cinco décadas de guerra. (Holguín, 2016)

Santos (2016) expreso "La Unión Europea es un socio fundamental para lograr un país en paz", esta aprobó un fondo fiduciario para el posconflicto, dirigido al desarrollo rural con proyectos productivos con un enfoque territorial. La primera de las líneas es el apoyo al programa Desarrollo Local Sostenible en zonas marginadas de Colombia, liderado por el Ministerio de Ambiente y Parques Nacionales. La canalización de recursos será la asistencia técnica a la Dirección de la Acción Integral contra Minas, organismo adscrito al Departamento Administrativo de la Presidencia. Y el cuarto mecanismo de entrega de recursos será una alianza con la Organización de las Naciones Unidas para la Alimentación y la Agricultura (FAO), para promover el fortalecimiento de la plataforma de diálogo social a nivel nacional y "mejorar las condiciones de tenencia y gobernanza de la tierra".

\section{Posconflicto desde la psicología social}

ohlberg (1984) expresó "el juicio moral se produce a partir de un
proceso de desarrollo en el que los individuos atraviesan una serie de
etapas que son idénticas para todos los seres humanos y tienen un orden preestablecido" (p.7). El análisis desde esta área es pertinente porque plantea una realidad desde la base del imaginario colectivo de la forma como ejecuta las relaciones interpersonales, propiamente. A partir de estos se posibilita cambiar la manera de ver y entender una realidad dentro de los resguardos, incluyendo ciudadanos como facilitadores que han estado con nexos con este escenario de vida, en especial los docentes del PECTI, como se explicará en detalle a continuación.

Para lograr esto se hace necesario, que existan unas personas que tomen la vocería desde adentro y que sean capacitados como formadores del posconflicto y para el mismo. Sí se objeta lo anterior se van a generar crisis al interior de los resguardos. Hay que tener presente que cada uno de ellos tiene un modelo 
de educación y que internamente se ciñen bajo unas políticas internas que a nivel constitucional se le conoce como legislación especial.

El Sistema Educativo Indígena, SEIP se viene construyendo a nivel nacional con los pueblos indígenas en Colombia, permite implementar el PECTI, develando la dinámica interna del resguardo, propiamente en los cabildos, se desarrolla la toma de decisiones entre el Gobernador y los cabildantes. El SEIP es el centro de la carta de navegación indígena, la mayoría de las decisiones se tomen con base a este, restándoles importancia a qué resguardo se pertenezca sino al hecho propio de ser indígena. El programa de educación cuenta con un equipo de asesores interdisciplinarios jurídicos, administradores, pedagogos, profesionales en el área de la gestión educativa, directivos docentes, lideres, lideresas y maestros indígenas. (Casiamo, 2005b).

El SEIP consolida la formación y actualización permanente del equipo de educación. Intercambio de experiencias educativas con otros pueblos indígenas y organizaciones sociales y populares. Participa en espacios interinstitucionales e interorganizativos de procesos educativos y establece mecanismos de regulación del comportamiento de los educadores y directivos docentes.

El SEIP tiene como objetivo consolidar el sistema educativo propio desde el fortalecimiento cultural, en relación con la construcción de la política pública de educación para los indígenas, implementando el proyecto educativo cultural territorial indígena PECTI pensamientos unidos, generando la promoción de la participación del joven en el proceso organizativo, como eje genera espacios de formación cultural desde la trasmisión del conocimiento ancestral. Didactización del PECTI, elaboración de materiales culturales. Además de los procesos propios de investigación, uso de la lengua materna y la tradición oral. Implementación del proyecto educativo cultural territorial indígena. Reflexión y formación pedagógica y educativa con comunidades, autoridades indígenas, educadores.

Coordinando dentro del aspecto social y en forma simultánea las aportaciones de la psicología social indicando lo relevante de la persona o grupo de personas anidadas desde un estímulo en común, en pro de alcanzar un logro colectivo, este último es fundamentalmente la protección de su identidad y de sus saberes ancestrales; consolidado con una aptitud motivada por el fortalecimiento de lo cognitivo. 
La educación, la salud y el saneamiento básico serán los principales detonantes de la bomba de tiempo que es Quibdó, a pesar de la educación, la salud y el saneamiento básico serán los principales detonantes de la bomba de tiempo que es Quibdó, a pesar de que el gobierno nacional destina anualmente 50.000 millones de pesos para esos sectores. Una de las situaciones más dramáticas en estos campos la viven los miles de indígenas desplazados que llegaron a la ciudad hace meses o años, y que no han recibido ninguna atención del Estado. (Calle, 2016).

El fortalecimiento del aspecto cognitivo permite la protección del saber ancestral, con respecto a lo afectivo brinda un valor culmen a la Tierra y la praxis de la toma de decisiones en el devenir del posconflicto teniendo presente que todo se debate entre los gobernadores de los cabildos y esta se proyectan a través del PECTI, fortalecimiento del aspecto conductual es la clave del éxito de la estrategia de la pedagogía crítica como estrategia de socialización, debido a que el aspecto conductual llevando al aspecto cognitivo permitiría que la tendencia de reaprender a comunicarse con lugares cargados de violencia se reacomoden como escenarios que posibiliten vivir en comunidad.

\section{Puntos de la mesa de negociaciones}

- as negociaciones entre las delegaciones del Gobierno Nacional y de la guerrilla de las FARC, y luego de haber superado 41 rondas de Inegociaciones en La Habana, las partes han logrado acuerdos parciales en cinco de los seis puntos de la agenda, descritos así: i) Una reforma agraria integral, ii) Participación en política por parte de las FARC, iii) narcotráfico y cultivos ilícitos, iv) Sometimiento a la justicia, v) Acuerdo definitivo sobre víctimas y, vi) Implementación, verificación y refrendación. Es evidente enmarcar la frase de perdón y olvido, para establecer un punto de partida ante la desolación, el desarraigo, el silencio y la impotencia que hoy se impregnan como forma de ethos impuesto violentamente, acabando la libertad cultural.

Una reforma agraria integral, no es viable sino se trastocan el fortalecimiento educativo enfatizando el aspecto cognitivo, afectivo y conductual a través del PECTI, esto es la clave del éxito de la estrategia de la pedagogía crítica como estrategia de socialización con base al aspecto conductual-cognitivo como se describió en asentamiento de psicología social, sino los esfuerzos serian efímeros. Ahora bien, la primera cuestión sería como organizaciones por ejemplo Fundación Ideas para la Paz, no se le permite hacer un mejor 
impacto en los resguardos y en vez de esto se le abre mayor espacio a la Iglesia Católica, posibilitando esta última un posible choque con las creencias e ideal colectivo de los mismos.

En cuanto a participación en política, postula los mismos aspectos anteriores forjando la cualificación de los líderes indígenas para una mayor sinergia y concreción del ciudadano-político. Seguidamente el narcotráfico y cultivos ilícitos no mermarán sino se practica el fortalecimiento del aspecto cognitivo, igualmente el sometimiento a la justicia; los dos últimos temas, el acuerdo definitivo sobre víctimas y, la implementación, verificación y refrendación son temas engañosos y es mejor optar con prudencia, a medida que se vaya avanzado en la implementación actual de las estrategias del postconflicto.

\section{Asentamiento filosófico}

T oucault (1976) explicitó la estructuración del biopoder, planteamiento 1 filosófico que explicita cómo se rige y se coarta la libertad de pensamiento, _ su accionar y la voluntad del ciudadano, en especial en la dimensión política a través de los limitantes socioeconómicos determinados por las políticas públicas, como es el caso de las limitantes en salud y la educación invasora en cierto sentido de la identidad cultural en los resguardos. Esta sinergia de biopoder evoluciona auscultando la dimensión de ciudadano político. (p. 20).

Biopoder es una nueva tecnología política, emergió en el corazón de la modernidad. Esta máquina fue denominada biopoder. En efecto, el poder asegura la preservación de los seres vivos. Lo ilimitado del poder de la vida sobre la vida, la posibilidad de la administración de los cuerpos y el cálculo dirigido de la vida, explican la asociación con los problemas del nacimiento, la longevidad, la salud pública y la migración. (Foulcault, 1976).

Además, el poder no es considerado como un objeto que el individuo cede al soberano (concepción contractual jurídico-política), sino que es una relación de fuerzas, una situación estratégica en una sociedad en un momento determinado. Por lo tanto, el poder, al ser resultado de relaciones de poder, está en todas partes. El sujeto está atravesado por relaciones de poder, no puede ser considerado independientemente de ellas. El poder, para Foucault, no solo reprime, sino que también produce: produce efectos de verdad, produce saber, en el sentido de conocimiento. 
Por ende, es necesario estudiar la vertebración del tejido social desde la pedagogía crítica para configurar la sociedad contemporánea que necesita organizarse, tener un desarrollo comunitario y llegar a vislumbrar y cristalizar el desarrollo social sostenible. El ideario filosófico sostiene en una construcción de mejores argumentos tamizados de diálogos dialogados, donde no exista la necesidad de hacer remembranza a la justicia transicional a cada paso. De esta manera, la paz, ya no es un concepto utópico sino un conjunto de acciones permitidas en una sociedad determinada la cual brinda la sociabilidad entre sus asociados. La paz, es el reto mayor para la construcción de un país.

En De la gramatología, Derrida (1967) indica que las sociedades avanzan en la medida que los conceptos que las sostienen son reevaluados constantemente, posibilitando una deconstrucción como sociedad, como individuos pero desde adentro, tal hipótesis abre paso a que existan educadores del postconflicto en cada resguardo para ser multiplicadores como conocedores de su ethos, la mos, en esencia de sus conceptos culturales. El pensamiento de Derrida y Freire llegan a encontrarse en este documento porque ambos proyectan la ruptura del sistema que los encadena y este no posee otro nombre que la religión, la universidad, la política y sus formas jerarquizas de poder. Por ende, era necesario describir el biopoder de Foucault pues el indígena no tiene la compresión occidental de libertad y de esclavitud.

Los indígenas estructuran su ideal de libertad en forma de comunicación con la naturaleza, muy diferentes a nuestro pensamiento, por ende el documento se puede leer en dos vías, una es la libertaria apoyada en el SEIP en cada resguardo y otra como forma de colonización, sí, no se da cumplimiento a la primera como está descrito. La deconstrucción semánticamente es viable para los resguardos pero no hay que olvidar que tanto Derrida como Freire apoyan sus ideologías en Occidente, situación que sería implantar un colonialismo occidental en contra del ideal de los resguardos, mas sin embargo la criticidad es propia del ciudadano que construye día a día una nueva sociedad, planteando en esencia que los resguardos pueden criticar como se va a desarrollar el posconflicto dentro de sus comunidades.

Abriendo paso a la estrategia de la pedagogía crítica como forma de socialización del posconflicto, el SEIP permite abrirse a esta realidad, conociendo la lucha y resistencia, después de un proceso de análisis, reflexión y debate entre las comunidades, líderes y autoridades, basados en los lineamientos político organizativo que pretenden hacerle frente a la grave situación de exterminio 
que padecen nuestras comunidades indígenas. La estrategia criticar desde su logos indígena, socializando los elementos del posconflicto a través de los lineamientos político organizativos del cabildo representado por cada Gobernador.

¿Para qué sirve la pedagogía crítica en el postconflicto?

La educación es un factor importante para la recuperación política y económica de un país, la nación necesitará capacitar a los docentes y acompañantes del PECTI para enfrentar los nuevos retos de la situación del posconflicto, desconocer el proceso desde la psicología social, el asentamiento ideológico filosófico, sociojurídico y, socioeconómico no es un garante para posibilitar una respuesta concertada del conflicto. En este orden de ideas, ingresa la pedagogía crítica, pues esta permite una intervención comunitaria más disertada, efectiva, disminuye los diálogos autoritarios y permite una mayor relación con los diálogos dialógicos, es la generación de una nueva voluntad del sujeto, una libertad de pensamiento que conlleva a desaprender y plantear soluciones viables y sostenibles en el tiempo.

"La pedagogía crítica es, por su parte, una propuesta de enseñanza que incita a los estudiantes a cuestionar y desafiar las creencias y prácticas que se les imparten. Consiste en un grupo de teorías y prácticas para promover la conciencia crítica. Muchos son los autores que han participado en el desarrollo y consolidación de la citada pedagogía crítica y entre ellos se encuentra el brasileño Paulo Freire. Una figura esta que se ha convertido en uno de los pensadores y teóricos de la enseñanza y de la educación más importantes de todos los tiempos." (Freire, 1970).

En los países que hayan sufrido de conflictos étnicos como el Congo, es muy importante revisar el currículo de las escuelas. Muchas veces, estos currículos están diseñados para discriminar grupos minoritarios; con el fin de lograr una paz duradera, es necesario eliminar estas fuentes de conflictos.

\section{Conclusiones}

T as aportaciones de la pedagogía crítica son diversas desde la óptica que se le desee plantear en el postconflicto, es de rescatar la interacción social y la educación, es la mayor argumentación social que faculta el proceso primario en el cual los individuos pueden intercambiar argumentos 
y desestabilizar sus creencias para reemplazarlas por otras nuevas, con el fin de proteger la identidad cultural del resguardo. La caracterización conlleva a un desarrollo moral permitiendo así una mejor integración de los factores desde la psicología social, analizando todo desde adentro, dialogando su ethos interno con la comunicación de lo externo cultural para cada resguardo.

Así, se logró dejar indicado en el planteamiento del Plan de Desarrollo de los Municipios de Carmen del Darién (Chocó) a través de la creación de un Director de Asuntos Étnicos, la protección específica de estos derechos indígenas. Esto disminuye las prácticas violentas que emergen de la forma de un razonamiento excluyente. La pedagogía crítica fortalece los lazos de fraternidad y de comunidad, donde el terror ha hecho presencia y ha imperado lo antihumano con ejercicios violentos, de autoritarismo y terror.

La paz necesita un hilo conductor, un análisis y una deconstrucción desde la forma de origen, la paz no propende por choques ideológicos de partidos políticos o movimientos, porque la paz es una construcción de base, donde cada uno de nosotros ha de hacer unas aportaciones de vida para la vida misma. No es un choque constante entre Santos y Uribe, es un choque de mi realidad y lo que deseo vivir a futuro. Centrándonos en esto, cada colombiano y colombiana debe comprender los esfuerzos colectivos que se aúnan y que se suman diariamente, donde el perdón es vital y la forma de ver el "mundo" en que vivimos, ya no del pasado sino del presente.

En síntesis, "la pedagogía crítica permite contrastar y generar la construcción de nuevos procesos concertados como ciudadano-víctimas y ciudadanovictimarios en relación con el Estado Social y Democrático de Derecho." Por ende hacer un pare reflexivo es vital, encaminado a la reconstrucción del tejido social mediante la pedagogía crítica propendiendo alcanzar: la paz.

\section{BIBLIOGRAFÍA}

Bolaños, E. (2016). Las propuestas de paz de los indígenas. [online] El Espectador. Available at: http://www.elespectador.com/noticias/paz/propuestas-de-paz-de-los-indigenas-articulo-392520 [Accessed 23 Jul. 2016].

Botero, D. (2016). [online] Aprendeenlinea.udea.edu.co. Available at: http://aprendeenlinea.udea. edu.co/revistas/index.php/unip/article/viewFile/13280/11899 [Accessed 23 Jul. 2016].

Calle, M. (2016). La hora más oscura de Quibdó: desplazamiento indígena. [online] Semana.com. Available at: http://www.semana.com/especiales/hora-mas-oscura-quibdo/desplazamientoindigena.html [Accessed 23 Jul. 2016]. 
Derrida, J. (1967). De la gramatología. Editorial Siglo XXI.

Freire. (1970). Pedagogía del oprimido. Editores Siglo XXI.

Foulcautl. (1976). Defender la sociedad. Barcelona. p. 11.

Gómez. (2016). [online] Available at: Memoria Histórica (2015). Citado en: http://WwW. centrodememoriahistorica.gov.co/descargas/informes2012/encuesta.pdf [Accessed 23 Jul. 2016].

Holguín. (2016). La Unión Europea anunció más ayuda para posconflicto. El Tiempo, [online] p. 7. Available at: http://www.eltiempo.com/ [Accessed 23 Jul. 2016].

Londoño. (2016). Mesa de negociaciones. [Online] www.mesadeconversaciones.com.co Available at: https://WwW.mesadeconversaciones.com.co/documentos/informes [Accessed 23 Jul. 2016].

Material original "Moral Stages: A Current Formulation and a Response to Critics", Lawrence Kohlberg, (S Karger Publisher, 1984).

Ministerio del Interior. (2016). Plan de salvaguarda de las comunidades embera, embera chamí, embera katío y embera dobida del área de juridicción del crich. Chocó: Elicio Caisamo, http://siic. mininterior.gov.co/sites/default/files/p.s_embera_organizacion_crich.pdf.

Ramirez. (2016). [online] Available at: http://institutodeestudiosurbanos.info/endatos/0100/0160/ docs/SISBEN.pdf [Accessed 23 Jul. 2016].

\section{Sentencia C-288 del 2012.}

Ulloa. (2016). Embera Chamí. [online] http://www.mincultura.gov.co/. Available at: http:// www.mincultura.gov.co/areas/poblaciones/noticias/Documents/Caracterizaci\%C3\%B3n\%20 del\%20pueblo\%20Embera\%20Cham\%C3\%AD.pdf [Accessed 23 Jul. 2016].

Valverde, J. (2016). Unidad para la Atención y Reparación Integral a las Víctimas. [online] URI. Available at: http://participaz.com/images/cartillas/reparaciones_cap7.pdf [Accessed 23 Jul. 2016]. 

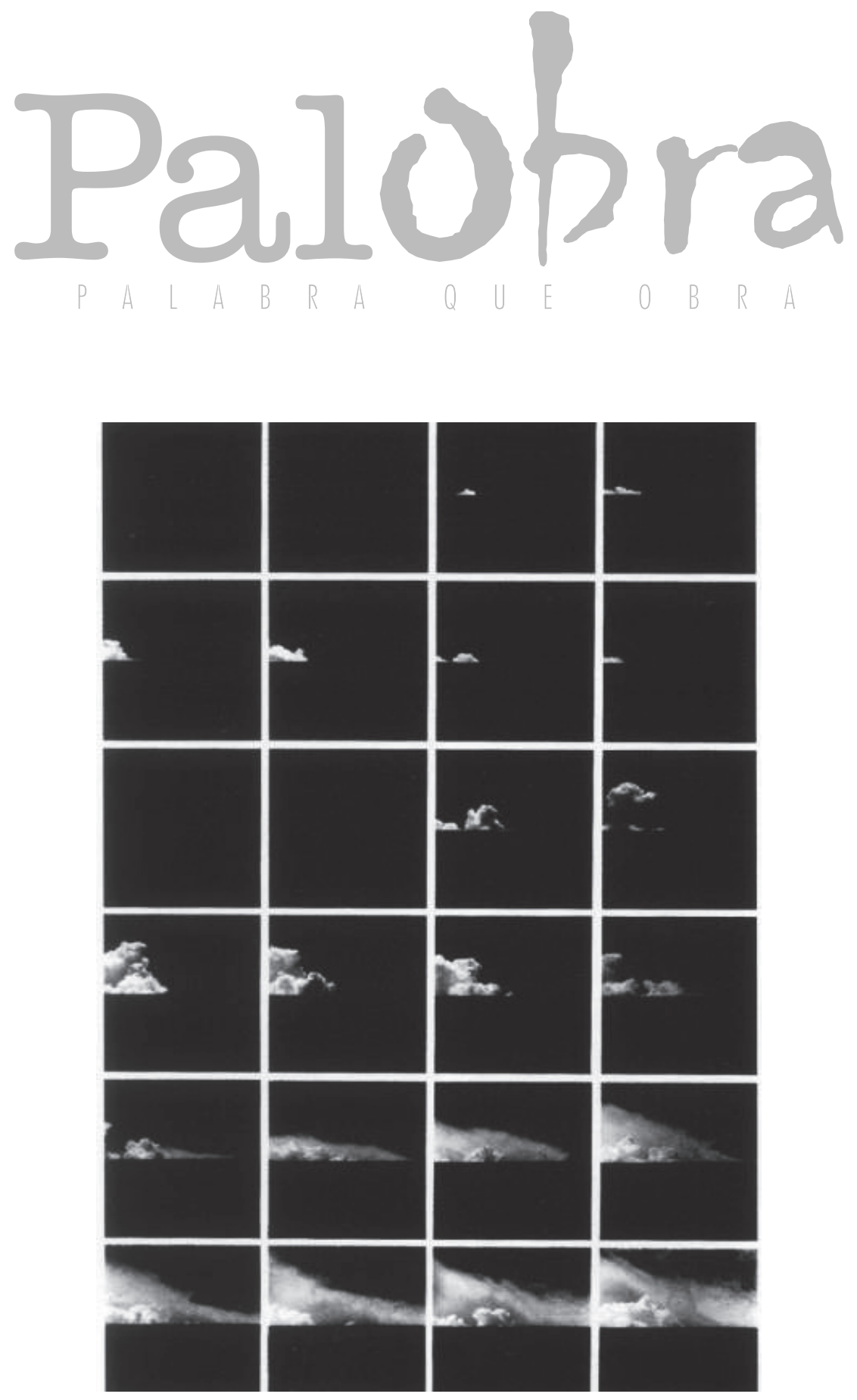

Boquerón. 1980.

Fotografía. 